PHYSICAL REVIEW A, VOLUME 63, 059901(E)

\title{
Erratum: Double-resonance frequency shift in a hydrogen maser [Phys. Rev. A 62, 063405 (2000)]
}

\author{
M. A. Humphrey, D. F. Phillips, and R. L. Walsworth
}

(Published 17 April 2001)

DOI: 10.1103/PhysRevA.63.059901

PACS number(s): 32.60.+i, 32.80.Wr, 99.10.+g

We wish to clarify an inconsistency in our notation where we have defined the term $\mathbf{H}_{C}$ in two different ways. In Sec. II, we have written the microwave cavity field as

$$
\mathbf{H}(\mathbf{r}, \omega)=\sqrt{4 \pi} p_{C}(\omega) \mathbf{H}_{C}(\mathbf{r})
$$

Here, the amplitude and time dependence of the field are included in $p_{C}(\omega)$, whereas $\mathbf{H}_{C}$ is merely an orthonormal vector describing the spatial variation of the field.

On the other hand, in Sec. III and Sec. IV, we have defined the microwave cavity field as

$$
\mathbf{H}(\mathbf{r}, t)=\mathbf{H}_{C}(\mathbf{r}) \cos (\omega t)
$$

Here, $\mathbf{H}_{C}$ represents the time-independent amplitude of the microwave cavity field. With this definition, the amplitude of the coupling between states $|2\rangle$ and $|4\rangle$ would be given by $H_{24}=\left\langle 2\left|\hat{\boldsymbol{\mu}} \cdot \mathbf{H}_{C}\right| 4\right\rangle$. The couplings to the dressed states $\left(H_{4 a}, H_{4 b}\right.$, and $\mathrm{H}_{4 c}$ ) are similarly defined. 